\title{
Delusional Disorder
}

National Cancer Institute

\section{Source}

National Cancer Institute. Delusional Disorder. NCI Thesaurus. Code C94379.

A disorder characterized by the presence of one or more nonbizarre delusions that persist for at least 1 month; the delusion(s) are not due to schizophrenia or a mood disorder, and do not impair psychosocial functioning apart from the ramifications of the delusion(s). 